\title{
VALUE DISTRIBUTION OF QUASIMEROMORPHIC MAPPINGS
}

\author{
SEPPO RICKMAN
}

\section{Introduction}

For some time it has been known that the right direction to extend the geometric parts of the theory of analytic functions in the plane to real $n$-dimensional space is given by the theory of quasiregular mappings. In this article we shall deal with recent results closely related to Nevanlinna theory, starting with a Picard type theorem on omitted values. In the light of these results the plane theory is an exceptional case due mainly to the separating property of arcs in plane domains. In spite of a striking contrast here between the dimensions $n=2$ and $n \geqq 3$ surprisingly strong results are true for higher dimensional mappings.

Let $G$ be a domain in the Euclidean $n$-space $R^{n}$ and let $f: G \rightarrow R^{n}$ be continuous. We say that $f$ is quasiregular if (1) $f \in W_{n \text {,loc }}^{1}(G)$, i.e. $f$ has distributional partial derivatives which are locally $L^{n}$-integrable, and (2) there exists $K, 1 \leqq K<\infty$, such that

$$
\left|f^{\prime}(x)\right|^{n} \leqq K J_{f}(x) \quad \text { a.e. }
$$

Here $f^{\prime}(x)$ is the formal derivative defined by means of the partial derivatives which by (1) exist a.e., $\left|f^{\prime}(x)\right|$ is its supremum norm, and $J_{f}(x)$ is the Jacobian determinant. For the purpose of this article we call a quasiregular mapping $K$-quasiregular if (1.1) is satisfied although this is not common terminology. The definition of quasiregularity extends immediately to maps $f: M \rightarrow N$ where $M$ and $N$ are Riemannian $n$-manifolds, see for example [5]. The term quasimeromorphic is reserved for the case where $M$ is a domain in $R^{n}$ or $\bar{R}^{n}=R^{n} \cup\{\infty\}$ and $N=\bar{R}^{n}$. The space $\bar{R}^{n}$ is equipped with the spherical metric. A quasiregular homeomorphism is called a quasiconformal mapping.

The theory of quasiregular mappings was initiated by Rešetnjak around 1966 in a series of papers and a systematic study was continued mainly by Martio, Rickman, and Väisälä some years later. For the basic theory we refer to $[3,4]$ and to the expository articles $[10,15]$.

If $n=2$, the 1-quasiregular mappings are exactly the analytic functions and any quasiregular mapping $f$ can be written as $f=g \circ h$ where $h$ is quasiconformal 
and $g$ analytic. If $n \geqq 3$, the 1-quasiregular mappings are Möbius transformations or constants, and it is essential to allow a distortion factor $K$ in order to obtain a class of mappings which is interesting in the function theoretic sense.

One of Rešetnjak's early main results is that a nonconstant quasiregular mapping is discrete and open. Hence these topological properties remain the same as in the plane. In 1967 Zorič [16] proved an important rigidity property for locally homeomorphic quasiregular mappings $f: R^{n} \rightarrow R^{n}$ for $n \geqq 3$, namely, that they are in fact homeomorphisms. This result was conjectured for a slightly smoother class of mappings by Lavrent'ev already in 1938. On the other hand, for every $n \geqq 3$ there exists $K_{n}>1$ such that every nonconstant $K_{n}$-quasiregular mapping is locally homeomorphic.

\section{The problem of Picard's theorem}

In [16] Zorič gave a simple example of a nonconstant quasiregular mapping $f: R^{3} \rightarrow R^{3} \backslash\{0\}$ and the construction can immediately be generalized for any $n \geqq 4$. It resembles the exponential function in the plane. In this connection Zoric also raised the question whether a Picard's theorem is true also in dimensions $n \geqq 3$ for quasiregular mappings. At a rather early stage it was proved in [4] and [8] that for a nonconstant quasiregular mapping $f: R^{n} \rightarrow R^{n}$ the set $\complement f R^{n}$ of omitted values is of zero conformal capacity. A few years ago the question of the existence of a Picard's theorem was answered, namely, the set of omitted values is in fact finite, more precisely as follows.

2.1. Theorem [11]. For each $n \geqq 3$ and each $K \geqq 1$ there exists a positive integer $q=q(n, K)$ such that if $f: R^{n} \rightarrow R^{n} \backslash\left\{a_{1}, \ldots, a_{q}\right\}$ is $K$-quasiregular and $a_{1}, \ldots, a_{q}$ are distinct points in $R^{n}$, then $f$ is constant.

It has been conjectured already from the appearance of Zorič's article [16] that the Picard's theorem is true in the same form for $n \geqq 3$ for quasiregular mappings as in the plane, i.e. no more than one point in $R^{n}$ can be omitted. However, this was disproved for $n=3$ recently and the result is that (at least for $n=3$ ) Theorem 2.1 is in fact qualitatively best possible, more precisely as follows.

2.2. Theorem [14]. For each positive integer $p$ there exists a nonconstant quasiregular mapping $f: R^{3} \rightarrow R^{3}$ which omits $p$ points.

The most important tool in the proofs in the theory of quasiregular mappings for dimensions $n \geqq 3$ is the method of moduli of path families. A potential theory exists in the form that the substitute for harmonic functions consists of extremals of certain elliptic variational integrals. The Euler equation of such a variational integral is a second order quasilinear partial differential equation and so the extremals are solutions of such equations. The use of variational techniques is important in Rešetnjak's early studies of quasiregular mappings. 
In the original proof of Theorem 2.1 in [11] both methods mentioned above were used. Here I will briefly outline a proof which uses only the method of path families. For more details, see [13].

2.3. Idea of proof of Theorem 2.1. Suppose that $f: R^{n} \rightarrow R^{n} \backslash\left\{a_{1}, \ldots, a_{q}\right\}$ is a nonconstant $K$-quasiregular mapping where $a_{1}, \ldots, a_{q}$ are distinct points in the ball $B(1 / 2)=\left\{x \in R^{n}|| x \mid<1 / 2\right\}$. We shall first consider a situation where $f$ behaves in a sense like a mapping of finite order. Let $n(E, y)$ be the number of points in $f^{-1}(y) \cap E$ with multiplicity regarded and let $v(E)=v(E, S)$ be the average of $n(E, y)$ when $y$ runs over the unit sphere $S$. Let a quasiconformal mapping $\varphi$ of $R^{n}$ onto itself be given and let $U$ be a Borel set such that $\varphi^{-1} B(1 / 2) \subset U \subset \varphi^{-1} \bar{B}(1 / 2)$. Set $W=\varphi^{-1} \bar{B}(2)$. Assume now that

$$
v(W) \leqq L v(U) .
$$

If we also assume that $v(U)$ is sufficiently large, it is possible (see $[9,4.1])$ to relate the average $v(U)$ and the average $v\left(X, S_{j}\right)$ over a small sphere $S_{j}=S\left(a_{j}, \sigma\right)$, where $X=\varphi^{-1} B(1)$, such that

$$
v\left(X, S_{j}\right) \geqq \frac{3}{4} v(U) .
$$

The radius $\sigma$ is chosen so that $v(U)=c_{1}(\log (1 / \sigma))^{n-1}$. Here $c_{1}, c_{2}, \ldots$ are positive constants which depend on $n$ and $K$ only. Set $Y=\varphi^{-1} B(3 / 2)$ and let $\Gamma_{j}$ be the family of paths connecting $f^{-1} S_{j}$ to $\bigcup_{k \neq j} f^{-1} S_{k}$ in $Y \backslash X$. Because each $a_{j}$ is omitted, each component of $f^{-1} B\left(a_{j}, \sigma\right)$ tends to $\infty$. Since we also have (2.5), it follows (see [12, (6.6)]) that we are able to find a $j$ such that the modulus $M\left(\Gamma_{j}\right)$ of $\Gamma_{j}$ satisfies $M\left(\Gamma_{j}\right) \geqq d(n) q^{1 /(n-1)}$ for some $d(n)>0$. On the other hand, each path in the image family $f \Gamma_{j}$ connects $S_{j}$ and $S\left(a_{j}, \sigma_{0}\right)$ where

$$
\sigma_{0}=\frac{1}{2} \min _{j \neq k}\left|a_{j}-a_{k}\right|
$$

Remembering that $\sigma$ is small we conclude that $M\left(f \Gamma_{j}\right)$ is small. But this is possible only if the average $v\left(Y, S\left(a_{j}, t\right)\right)$ is large for some $t \in\left[\sigma, \sigma_{0}\right]$. If we put all these quantitatively together and relate averages over different spheres as we did in (2.5), we end up with an estimate (see $[12,6.7]$ for the technique)

$$
v(W) \geqq c_{2} q^{1 /(n-1)}(\log (1 / \sigma))^{n-1} .
$$

By the choice of $\sigma$ we get $v(W) \geqq c_{3} q^{1 /(n-1)} v(U)$ which with (2.4) gives

$$
q \leqq L^{n-1} c_{3}^{1-n} \text {. }
$$

The proof is now completed by finding a pair $U, W$ so that (2.4) holds and $v(U)$ is large. Write $v(r)=v(\bar{B}(r))$, and for $v(r) \geqq 1$ define

$$
r^{\prime}=r+\frac{r}{v(r)^{\alpha}}, \quad \alpha=\frac{1}{2(n-1)} .
$$


By using ideas which go back to E. Borel we can find a set $E \subset[1, \infty[$ of finite logarithmic measure and

$$
v\left(r^{\prime}\right) \leqq 2 v(r) \text { if } \quad r \in[1, \infty[\backslash E .
$$

Let $r \in\left[1, \infty\left[\backslash E\right.\right.$. We write the ball $\bar{B}(r)$ as a finite disjoint union of sets $U_{i}$ such that for some $K(n)$-quasiconformal map $\varphi_{i} \varphi_{i}^{-1} B(1 / 2) \subset U_{i} \subset \varphi_{i}^{-1} \bar{B}(1 / 2)$ and so that the sizes of the sets $U_{i}$ are within fixed bounds measured in the hyperbolic metric of the ball $B\left(r^{\prime}\right)$. We may choose the $\varphi_{i}$ so that the sets $W_{i}=\varphi_{i}^{-1} \bar{B}(2)$ are contained in $\bar{B}\left(r^{\prime}\right)$ and do not overlap more than $b(n)$ times. It then follows from (2.7) and the fact that $v(r) \rightarrow \infty$ as $r \rightarrow \infty$ that if $r$ is sufficiently large, then there exists an index $i$ such that (2.4) holds for the pair $U_{i}, W_{i}$ with $L=L(n)$ and $v\left(U_{i}\right) \geqq$ $v(r)^{1 / 4}$. The pair $U_{i}, W_{i}$ has then the desired properties. That $a_{1}, \ldots, a_{q}$ were assumed to lie in $B(1 / 2)$ is no restriction because this can always be achieved by a preliminary Möbius transformation.

2.8. Remark. Because of the localizing method in the proof of Theorem 2.1, we obtain by the same proof also a result which corresponds to the big Picard's theorem [11, Theorem 1.2].

It is interesting to see how the idea of the given proof works in the plane to give the sharp result, namely Picard's theorem. We did not use the fact that $\infty$ is omitted. Let therefore $f: R^{2} \rightarrow \bar{R}^{2} \backslash\left\{a_{1}, a_{2}, a_{3}\right\}$ be a nonconstant $K$-quasimeromorphic mapping where $a_{1}, a_{2}, a_{3}$ are distinct points in $B(1 / 2)$. Let $U, W$ be the pair $U_{i}, W_{i}$ chosen at the end of the proof. Let $\gamma_{y}^{j}:[0,1] \rightarrow \bar{B}(1)$ be the linear path $\gamma_{y}^{j}(t)=t y+(1-t) a_{j}$. By the method of the proof of $[9,4.1]$ we can find $y \in S$ such that $n(U, y) \geqq v(U) / 2$ and there exists at least $3 n(U, y) / 4$ maximal lifts $\alpha_{x}^{j}$ of $\gamma_{y}^{j}$ such that $\alpha_{x}^{j}(1)=x \in U, \alpha_{x}^{j}(\sigma / 2) \in X$. But then there exists at least $n(U, y) / 4$ points $x \in U$ with such a lift $\alpha_{x}^{j}$ for every $j=1,2,3$. Each triple $\left(\alpha_{x}^{1}, \alpha_{x}^{2}, \alpha_{x}^{3}\right)$ of such lifts divides the plane into three Jordan domains $D_{x}^{1}, D_{x}^{2}, D_{x}^{3}$. Consider another triple $\left(\alpha_{z}^{1}, \alpha_{z}^{2}, \alpha_{z}^{3}\right)$ and the domains $D_{z}^{j}, z \neq x$. We may assume that the segment $\left|\gamma_{y}^{j}\right|=\left[a_{j}, y\right]$ does not touch the image of the branch set. It follows that the paths $\alpha_{x}^{j}$ and $\alpha_{z}^{j}$ are disjoint and that two of the domains $D_{z}^{j}$ must be contained in one of the domains $D_{x}^{1}, D_{x}^{2}, D_{x}^{3}$. This has the effect of squeezing some of the domains when $v(U)$ is tending to $\infty$. But this implies that $M\left(\Gamma_{j}\right)$ gets arbitrarily large for some $j$ when increasing $r$. This is impossible according to the proof in 2.3.

The essential fact that makes the proof work for three points omitted in $\bar{R}^{2}$ is that paths have an effective separating property in the plane. In dimension three paths do not separate, and even more, tubular neighborhoods of paths do not separate in general. The construction in the proof of Theorem 2.2 depends on this latter fact. In contrast to Zorič's example where one point in $R^{3}$ is omitted, the construction of a nonconstant quasiregular mapping of $R^{3}$ omitting $p \geqq 2$ points in $R^{3}$ is complicated and one can even show that in a sense this must be so. It is probable that Theorem 2.2 is true also for $n \geqq 4$. 


\section{A defect relation}

Nevanlinna's second main theorem [6] for meromorphic functions is a far reaching extension of Picard's theorem. In this section we shall discuss what is known in this direction for quasimeromorphic mappings in dimensions $n \geqq 3$. For simplicity let us consider only the case of a nonconstant $K$-quasimeromorphic mapping $f: R^{n} \rightarrow \bar{R}^{n}$ and $n \geqq 2$. As in the classical theory let $n(r, y)$ be $n(\bar{B}(r), y)$, i.e. the number of points in $f^{-1}(y) \cap \bar{B}(r)$ with multiplicity regarded. We call $n(r, y)$ the counting function. Let $A(r)$ be the average of $n(r, y)$ over $\bar{R}^{n}$ with respect to the spherical metric.

If for some point $a \in \bar{R}^{n}$ and some $r>0,1-n(r, a) / A(r)>0$, then $a$ is covered less than in average by the restriction $f \mid \vec{B}(r)$. The positive part $(1-n(r, a) / A(r))_{+}$ can therefore be called the defect at $a$ in $\bar{B}(r)$.

Let for the moment $n=2$ and $f: R^{2} \rightarrow \bar{R}^{2}$ be meromorphic. Nevanlinna's second main theorem implies that there exists an exceptional set $F \subset[0, \infty[$ of finite measure such that

$$
\limsup _{\substack{r \rightarrow \infty \\ r \notin F}} \sum_{j=1}^{q}\left(1-\frac{N\left(r, a_{j}\right)}{T(r)}\right) \leqq 2
$$

whenever $a_{1}, \ldots, a_{q}$ are distinct points in $\bar{R}^{2}$. Here $N(r, a)$ and $T(r)$ are logarithmic integrals of $n(r, a)$ and $A(r)$ respectively. The inequality (3.1) gives immediately Nevanlinna's defect relation

$$
\sum_{a \in \bar{R}^{2}} \delta(a) \leqq 2
$$

where

$$
\delta(a)=1-\limsup _{r \rightarrow \infty} \frac{N(r, a)}{T(r)}
$$

is the Nevanlinna defect at $a$.

A theory parallel to Nevanlinna's was created by Ahlfors [1] where a highly geometric point of view is taken. The result in Ahlfors's theory corresponding to (3.1) is the following (see [7, p. 350]): There exists an exceptional set $E \subset[1, \infty[$ of finite logarithmic measure such that

$$
\limsup _{\substack{r \rightarrow \infty \\ r \notin E}} \sum_{j=1}^{q}\left(1-\frac{n\left(r, a_{j}\right)}{A(r)}\right)_{+} \leqq 2
$$

whenever $a_{1}, \ldots, a_{q}$ are distinct points in $\bar{R}^{2}$. Thus the sum of the defects at $a_{1}, \ldots, a_{q}$ in $\bar{B}(r)$ is asymptotically bounded by 2 outside the exceptional set. Ahlfors himself pointed out in [1] that his theory works for quasimeromorphic mappings in the plane as well. This is the case for parts of Nevanlinna's theory too; for example, 
a second main theorem has been proved by af Hällström by using Ahlfors's theory as a starting point.

For quasimeromorphic mappings in dimensions $n \geqq 3$ a result connected to (3.4) has been proved in the following form.

3.5. Theorem [12]. Let $n \geqq 3$ and let $f: R^{n} \rightarrow \bar{R}^{n}$ be a nonconstant $K$-quasimeromorphic mapping. Then there exists a set $E \subset[1, \infty[$ of finite logarithmic measure and a constant $C(n, K)<\infty$ depending only on $n$ and $K$ such that

$$
\limsup _{\substack{r \rightarrow \infty \\ r \notin E}} q\left[\frac{1}{q} \sum_{j=1}^{q}\left(1-\frac{n\left(r, a_{j}\right)}{A(r)}\right)_{+}\right]^{n-1} \leqq C(n, K)
$$

whenever $a_{1}, \ldots, a_{q}$ are distinct points in $\bar{R}^{n}$.

Theorem 2.1 is clearly a corollary of Theorem 3.5. By Theorem 2.2 the bound $C(n, K)$ is qualitatively sharp at least for $n=3$. The left hand side of (3.6) reduces to that of (3.4) for $n=2$. However, it is an open question whether the exponent $n-1$ is the best in (3.6). There seems to be some indication that it could possibly be 1 as in (3.4). The proof of Theorem 3.5 consists of a careful analysis of lifts of paths together with a generalization of the method of moduli of path families. The very basic idea is in the spirit of the proof of Theorem 2.1 outlined in 2.3.

\section{Averages of the counting function}

For meromorphic functions Nevanlinna's second main theorem bounds the ratios $N(r, a) / T(r)$ from below. The inequality (3.1) is an example of this. In the other direction Nevanlinna's first main theorem implies that

$$
N(r, a) \leqq T(r)+O(1)
$$

where the $O(1)$ depends on $a$. The relationship between $T(r)$ and averages of $N(r, y)$ with respect to measures in $\bar{R}^{2}$ is discussed in [7, pp. 177-182]. Ahlfors's wellknown covering theorems $[1$, p. 164, 165] are examples of average results for the counting function $n(r, y)$.

For quasiregular mappings a general result for averages of the counting function $n(r, y)$ has been given in [5]. In [5] we consider a quasiregular mapping $f: M \rightarrow N$ where $M$ and $N$ are connected Riemannian $n$-manifolds, $M$ noncompact and $N$ compact. To avoid the discussion of exhaustions of $M$ we will here present the result for $M=R^{n}$. We consider measures $\mu$ in $N$ such that Borel sets are measurable, $0<\mu(N)<\infty$, and for any ball $B(x, r)$ in $N$

$$
\mu(B(x, r)) \leqq h(r)
$$


holds, where $h$ is an increasing, continuous, and positive function such that

$$
\int_{0}^{1} \frac{h(\varrho)^{1 / p n}}{\varrho} d \varrho<\infty
$$

for some $p>2$. Let $v_{\mu}(r)$ be the average of the counting function $n(r, y)$ with respect to $\mu$. Our result is then that outside an exceptional set $v_{\mu}(r)$ is asymptotically near the average $A(r)$ with respect to the Lebesgue measure of $N$, more precisely as follows.

4.2. Theorem [5, Theorem 5.11(1)]. If $f: R^{n} \rightarrow N$ is a nonconstant quasiregular mapping, there exists a set $E \subset[1, \infty[$ of finite logarithmic measure such that for any measure $\mu$ in $N$ as above we have

$$
\lim _{\substack{r \rightarrow \infty \\ r \notin E}} \frac{v_{\mu}(r)}{A(r)}=1
$$

For meromorphic functions Hinkkanen [2] sharpened the result of 4.2 to the form that (4.1) can be replaced by

$$
\int_{0}^{1} \frac{h(\varrho)}{\varrho} d \varrho<\infty .
$$

\section{References}

[1] AhLFoRs, L.: Zur Theorie der Überlagerungsflächen. - Acta Math. 65, 1935, 157-194.

[2] HinkKanen, A.: On the averages of the counting function of a meromorphic function. - Ann. Acad. Sci. Fenn. Ser. A I Math. Dissertationes 26, 1980, 1-31.

[3] Martio, O., S. Rickman, and J. VÄISÄLÄ: Definitions for quasiregular mappings. - Ann. Acad. Sci. Fenn. Ser A I Math. 448, 1969, 1-40.

[4] MARTIO, O., S. RICKMAN, and J. VÄISÄLÄ: Distortion and singularities of quasiregular mappings. - Ann. Acad. Sci. Fenn. Ser A I Math. 465, 1970, 1-13.

[5] Mattila, P., and S. Rickman: Averages of the counting function of a quasiregular mapping. Acta Math. 143, 1979, 273-305.

[6] Nevanlinna, R.: Zur Theorie der meromorphen Funktionen. - Acta Math. 46, 1925, 1-99.

[7] Nevanlinna, R.: Analytic functions. - Springer-Verlag, Berlin-Heidelberg-New York, 1970.

[8] ReŠETnJAK, JU. G.: Extremal properties of mappings with bounded dístortion. - Sibirsk. Mat. Ž. 10, 1969, 1300-1310 (Russian).

[9] Rickman, S.: On the value distribution of quasimeromorphic maps. - Ann. Acad. Sci. Fenn. Ser. A I Math. 2, 1976, 447-466.

[10] Rickman, S.: On the theory of quasiregular mappings. - Proceedings of the Rolf Nevanlinna Symposium on Complex Analysis, Silivri 1976, 79-87.

[11] Rickman, S.: On the number of omitted values of entire quasiregular mappings. - J. Analyse Math. 37, 1980, 100-117.

[12] Rickman, S.: A defect relation for quasimeromorphic mappings. - Ann. of Math. (2) 114, 1981, 165-191. 
[13] Rickman, S.: Value distribution of quasiregular mappings. - Proceedings of the $18^{\text {th }}$ Scandinavian Congress of Mathematicians 1980, Progress in Mathematics Vol. 11, 1981, 430-440.

[14] Rickman, S.: The solution to the problem of Picard's theorem for quasiregular mappings in dimension three. - In preparation.

[15] VÄIs⿱̈亡̈̈̈, J.: A survey of quasiregular maps in $R^{n}$. - Proceedings of the International Congress of Mathematicians, Helsinki 1978, 685-691.

[16] ZoRIČ, V. A.: The theorem of M. A. Lavrentiev on quasiconformal mappings of space. - Mat. Sb. 74, 1967, 417-433 (Russian).

\author{
University of Helsinki \\ Department of Mathematics \\ SF-00100 Helsinki 10 \\ Finland
}

Received 6 May 1981 